\title{
Forecasting by Dynamic Regression Models with an Application
}

Hassan M. Alias (PhD)

Assistant Professor

Department of Statistics

University of Mosul

\author{
Nawal M. Al - Miamary \\ Assistant Lecturer \\ Department of Management Information Systems \\ University of Mosul
}

\begin{abstract}
Dynamic Regression Model is that model which takes the time into account. The modeling of the Dynamic Regression shows how the output is resulted from the input. This depends on the following:

1. The relation of the lag time with the input and output.

2. The time composition for the turbulence series (random error)

In order provide mathematical model, the relative model was identified by specifying the linear transformation function. The relative model of the transformation function was of the degree $(0,0,1)$. When the values of turbulence series were examined by using auto correlation and partial auto correlation coefficients, it is found that all of the coefficients were insignificant and that consequently proves the turbulence series which is a series of random residuals, so that: $\mathrm{N}_{\mathrm{t}}=\mathrm{a}_{\mathrm{t}}$.
\end{abstract}


إن استخدام موضوع السلاسل الزمنية في وصف الظاهرة لمعرفة طبيعة التغيرات التي تطرأ عليها في الفترات الزمنية وما ستكون عليه من المواضية اضنيع

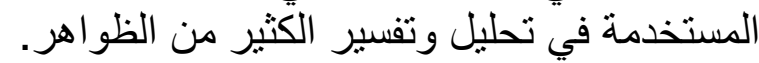

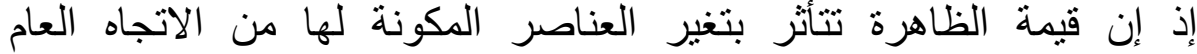

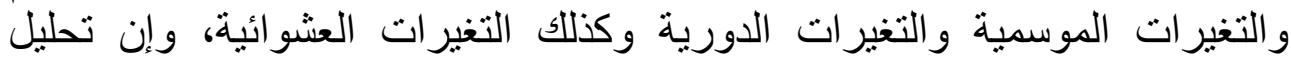

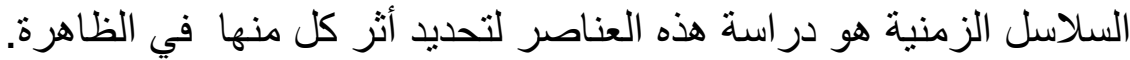

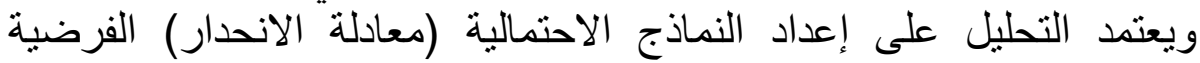

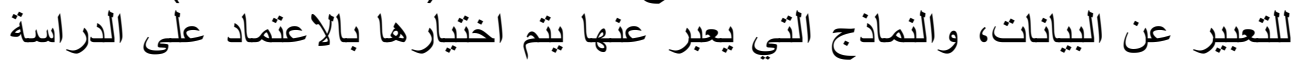

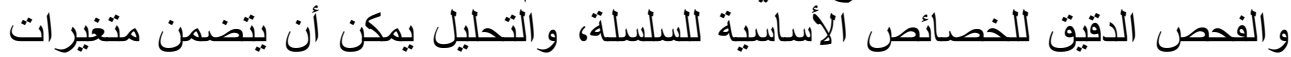
تفسيرية بالنسبة للنماذج المقتر حة. وإن استخدام أنموذج الانحدار الخطي في هذه الحال فئرة غير صحيح بسبب وجود

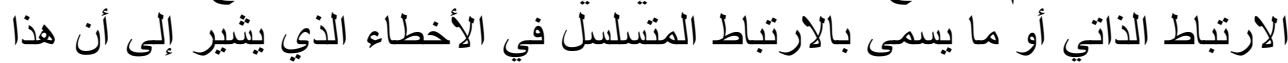

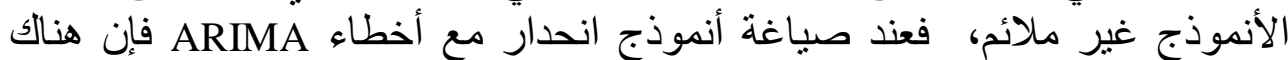

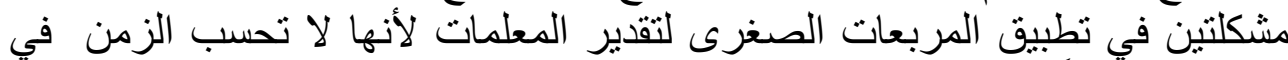

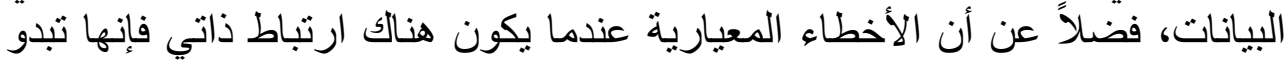

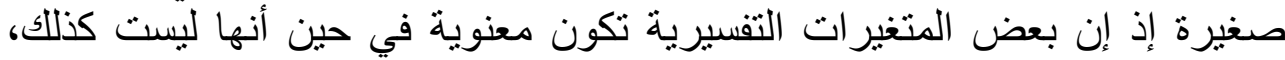

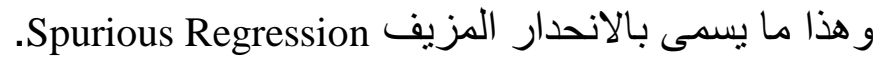

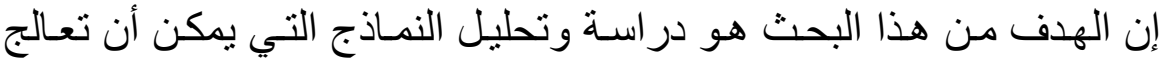

اللهدف

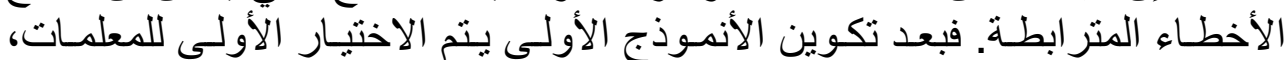

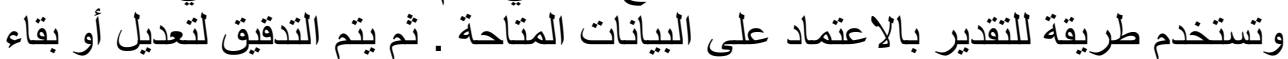

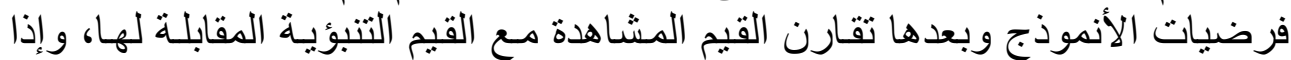

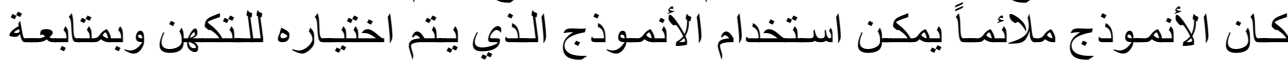

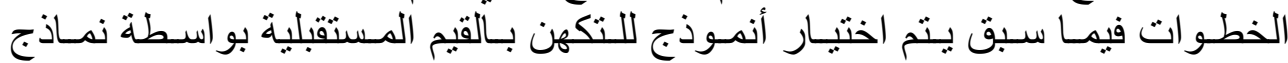

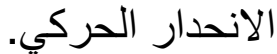

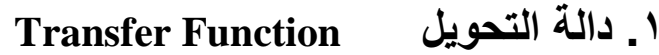

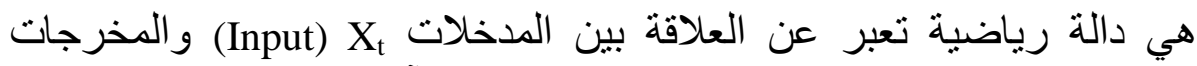

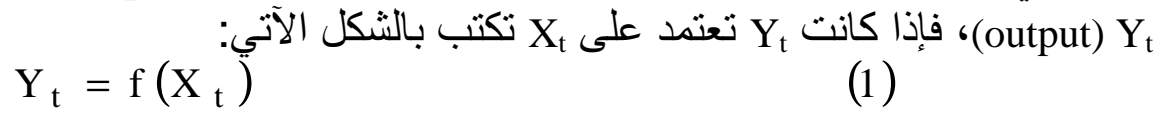

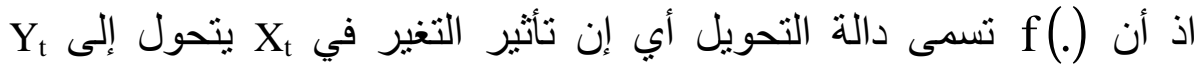
بطريقة ما محددة بواسطة الدالة (. التي يمكن التعبير عنها بأنها توصف الته العلاقة بين المدخلات والمخرجات. (Pankratiz,1991) (Jenkins and Box,1976,148) 
$(r \cdot)^{9} \cdot$

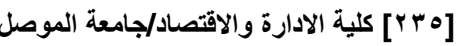

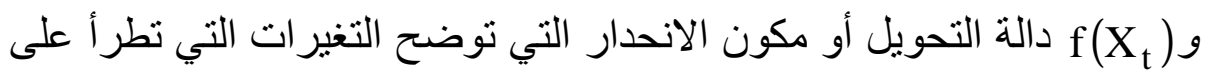

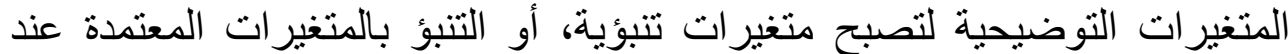

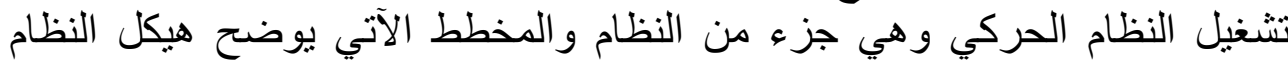
$\mathrm{X}_{\mathrm{t}} \longrightarrow\left(\begin{array}{r}\text { Input } \\ \text { Transfer } \\ \text { Function }\end{array}\right) \stackrel{\text { Ouput }}{\longrightarrow} \mathrm{Y}_{\mathrm{t}}$

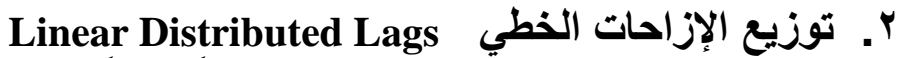
و بوصفها تركيباً خطياً من القيم الحالية و السابقة لـ f $\mathrm{Y}_{\mathrm{t}}=\mathrm{v}_{0} \mathrm{X}_{\mathrm{t}}+\mathrm{v}_{1} \mathrm{X}_{\mathrm{t}}+\mathrm{v}_{2} \mathrm{X}_{\mathrm{t}}+\cdots$

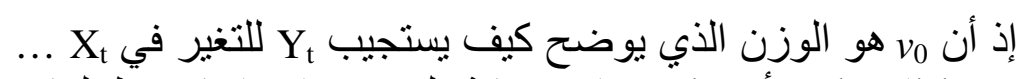

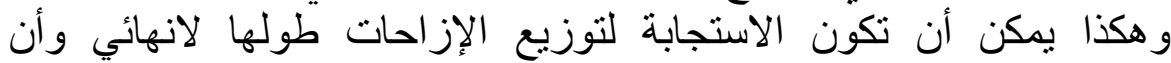
الأوزان (v) يمكن أن تكون موجبة أو سالبة (Pankratiz,1991, 149).

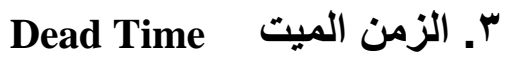

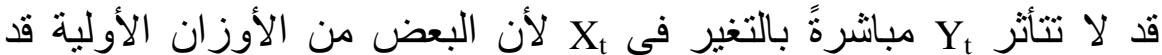

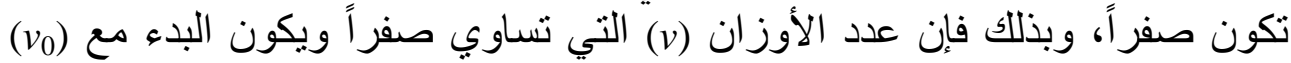

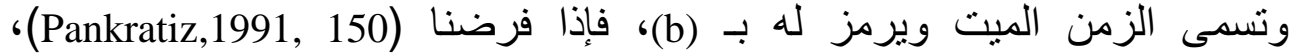

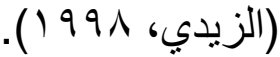

$$
\begin{aligned}
\mathrm{v}_{0}=\mathrm{v}_{1}=\mathrm{v}_{2} \\
\mathrm{v}_{3} \neq 0 \\
\mathrm{~b}=3
\end{aligned}
$$

أي أن bمثل معلمـة التأخير الزمني المتمثلـة بوقت الإزاحة الفعليـة المنقضي

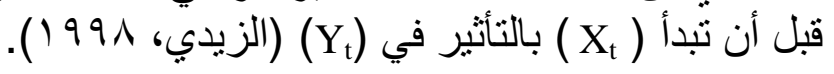

Impulse Response Function ـ دالة الاستجابة المحفزة (النبضية) عند إعادة كتابة دالة التحويل لتوزيع الإزاحات الخطية باتية باستخدام عامل

$$
\begin{aligned}
& \mathrm{Y}_{\mathrm{t}}=\mathrm{v}(\mathrm{B}) \mathrm{X}_{\mathrm{t}} \\
& \text { الإز احة الخلفية ومن خلال تعريف (B) v وتعويضها في ب ينتج: }
\end{aligned}
$$




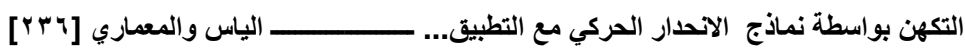

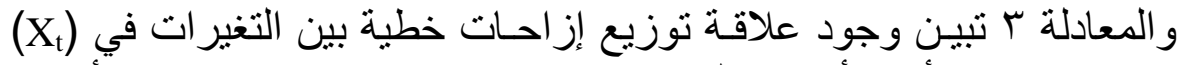
و التغيرات في (Yt) وأن الأوز الاستجابة المحفزة، والمجموعة الكاملة من الأوزان v تسمى بدالة الاستجابة المحفزة

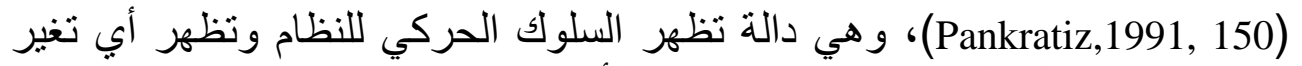

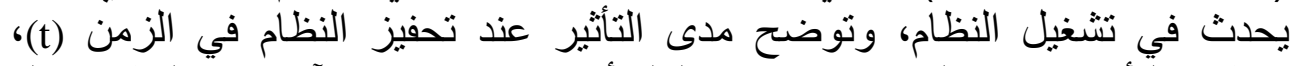

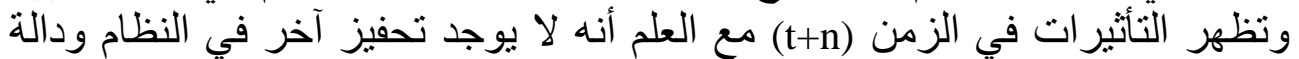
الاستجابة المحفزة هي مقياس لإستجابة المخرجات عند إجراء تغيرات التهري للمدخلات

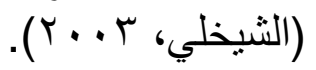

•ـ الهبوط الأسي لثلأوزان Exponential v-Weight Decay

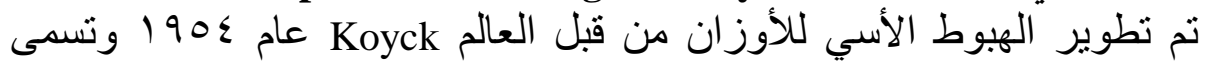

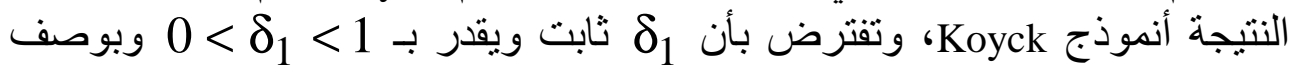

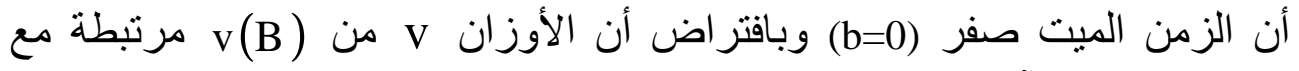

$$
\begin{aligned}
& \mathrm{v}_{1}=\delta_{1} \mathrm{v}_{0} \\
& \mathrm{v}_{2}=\delta_{1} \mathrm{v}_{1}
\end{aligned}
$$$$
\mathrm{v}_{\mathrm{k}}=\delta_{1} \mathrm{v}_{\mathrm{k}-1}
$$$$
\text { وبمعرفة v و }
$$$$
\mathrm{v}_{2}=\delta_{1} \mathrm{v}_{1}=\delta_{1}^{2} \mathrm{v}_{0}
$$$$
\mathrm{v}_{3}=\delta_{1} \mathrm{v}_{2}=\delta_{1}^{3} \mathrm{v}_{0}
$$

$$
\mathrm{v}_{\mathrm{k}}=\delta_{1}^{\mathrm{k}} \mathrm{v}_{0} \quad, \mathrm{k} \geq 0
$$

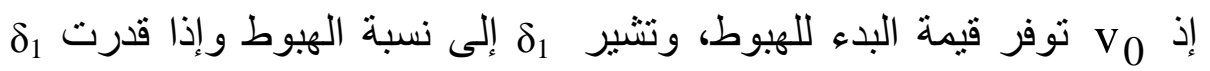
و v vيكون هناك استخدام جيد ومناسب للبيانات وبعدها يتم إيجاد تقدير

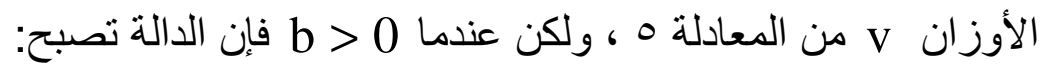

(Pankratiz,1991, 151)

$$
\mathrm{v}_{\mathrm{k}}=\delta_{1}^{\mathrm{k}-\mathrm{b}} \mathrm{v}_{\mathrm{b}} \quad, \mathrm{k}>\mathrm{b}
$$


$(r \cdot)^{9} \cdot$

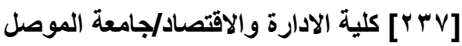

\section{صيغة العجز الاقتصادي من أنموذج Koyck}

\section{A parsimonious Form of the Koyck Model}

تستخدم طريقة Koyck لإيجاد صيغة لدالة التحويل لها معلمتان

$$
\begin{aligned}
& \text { وذللك باستبدال الأوزان (v)في (3) بـ ve } \\
& \mathrm{Y}_{\mathrm{t}}=\mathrm{v}_{0} \mathrm{X}_{\mathrm{t}}+\delta_{1} \mathrm{v}_{0} \mathrm{X}_{\mathrm{t}-1}+\delta_{1}^{2} \mathrm{v}_{0} \mathrm{X}_{\mathrm{t}-2}+\cdots \\
& \text { و الاستجابة لـ Y Yمكن إيجادها بخطو تين من (7) وكالآتي: } \\
& \text { الخطوة الأولى: بالتعويض عن (t) بـ (t-1) نحصل: } \\
& \mathrm{Y}_{\mathrm{t}-1}=\mathrm{v}_{0} \mathrm{X}_{\mathrm{t}-1}+\delta_{1} \mathrm{v}_{0} \mathrm{X}_{\mathrm{t}-2}+\delta_{1}^{2} \mathrm{v}_{0} \mathrm{X}_{\mathrm{t}-3}+\cdots
\end{aligned}
$$

الخطوة الثانية: بضرب طرفي المعادلة (8) في 1 (م)

$$
\begin{gathered}
\delta_{1} \mathrm{Y}_{\mathrm{t}-1}=\delta_{1} \mathrm{v}_{0} \mathrm{X}_{\mathrm{t}-1}+\delta_{1}^{2} \mathrm{v}_{0} \mathrm{X}_{\mathrm{t}-2}+\delta_{1}^{3} \mathrm{v}_{0} \mathrm{X}_{\mathrm{t}-3}+\cdots \\
\mathrm{Y}_{\mathrm{t}}=\mathrm{v}_{0} \mathrm{X}_{\mathrm{t}}+\delta_{1} \mathrm{Y}_{\mathrm{t}-1}
\end{gathered}
$$

وبالحصول على معلمـات قليــة يـتـ إعـداد نمـوذج للتقدير أكثر كفـاءة

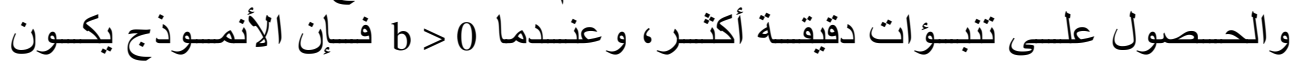
(Makridakis and Whelwright,1978)، (Pankratiz,1991, 153)

$$
\mathrm{Y}_{\mathrm{t}}=\mathrm{v}_{\mathrm{b}} \mathrm{X}_{\mathrm{t}-\mathrm{b}}+\delta_{1} \mathrm{Y}_{\mathrm{t}-1}
$$

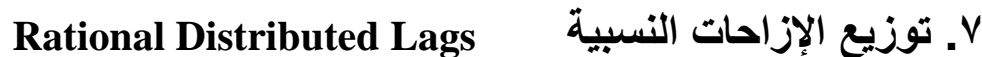

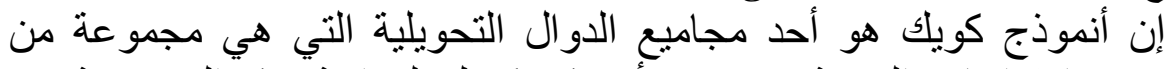

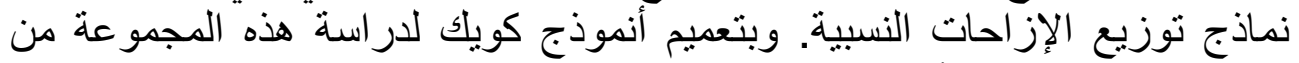
الدوال التحويلية. ومن أنموذج كويك في المعادلة ـ ( (Pankratiz,1991, 156):

$$
\begin{aligned}
& \left(1-\delta_{1} \mathrm{~B}\right) \mathrm{Y}_{\mathrm{t}}=\mathrm{v}_{0} \mathrm{X}_{\mathrm{t}} \\
& \mathrm{Y}_{\mathrm{t}}=\frac{\mathrm{v}_{0}}{1-\delta_{1} \mathrm{~B}} \mathrm{X}_{\mathrm{t}}
\end{aligned}
$$

وتعد المعادلة r ا معادلة متعددة الحدود النسبية لأن V(B) هي نسبة، ومنها

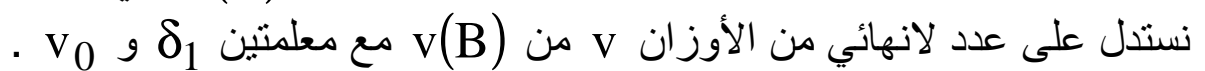

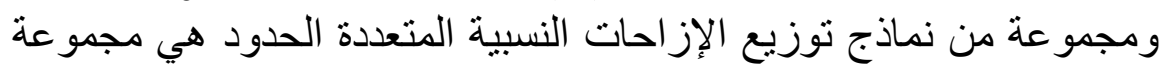

من دو ال الاستجابة المحفزة (B) المعطاة بو اسطة (Pankratiz,1991, 162):

$$
v(B)=\frac{\left.w^{(B)}\right) B^{d}}{\delta(B)}
$$




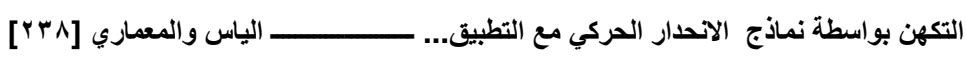

$$
\begin{aligned}
& \mathrm{w}(\mathrm{B})=\mathrm{w}_{0}+\mathrm{w}_{1} \mathrm{~B}^{1}+\mathrm{w}_{2} \mathrm{~B}^{2}+\ldots+\mathrm{w}_{\mathrm{h}} \mathrm{B}^{\mathrm{h}} \\
& \delta(\mathrm{B})=1-\delta_{1} \mathrm{~B}^{1}-\delta_{2} \mathrm{~B}^{2}-\cdots-\delta_{\mathrm{r}} \mathrm{B}^{\mathrm{r}} .
\end{aligned}
$$

رنبة h

$\delta$ رتبة ر رتبة

A. إيجاد الأوزان v من الأوزان

\section{Finding v Weight from w and $\delta$ Weights}

إذا كانت الأوزان w و م معروفة للأنموذج المقدر في الصيغة النسبية ويحتاج

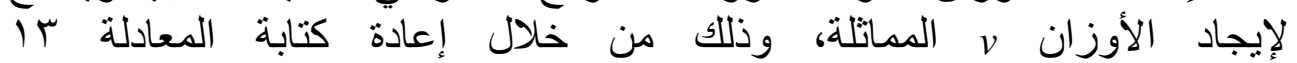

$$
\begin{aligned}
& \delta(\mathrm{B}) \mathrm{v}(\mathrm{B})={ }_{\mathrm{w}}(\mathrm{B}) \mathrm{B}^{\mathrm{d}} \\
& \mathrm{v}_{\mathrm{j}}=0, \\
& \text { ومنه سوف تتكون العلاقات الآتية (الزيدي، } \\
& v_{j}=\sum \delta_{j} v_{j-i}+w_{j-b} \\
& , j \geq b
\end{aligned}
$$

$w_{j-b}=0$

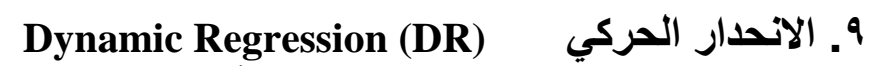

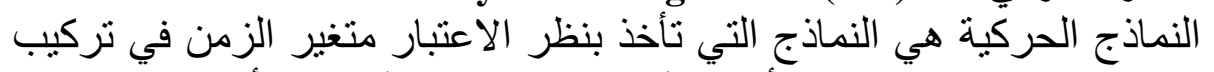

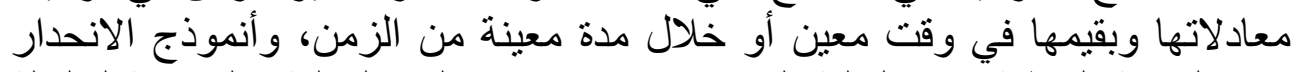

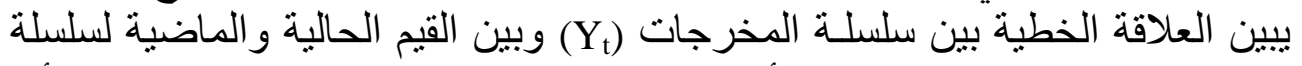
المدخلات ...2

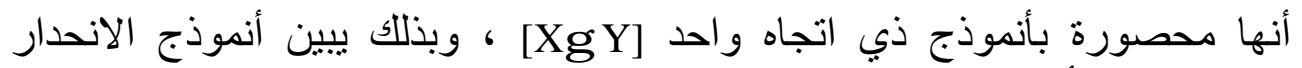

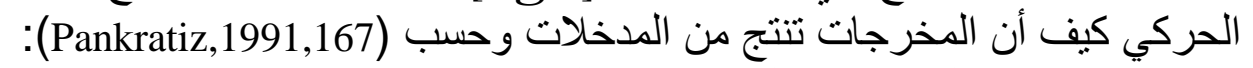

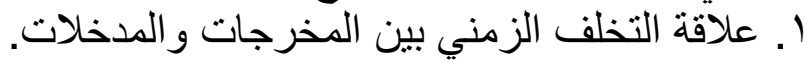

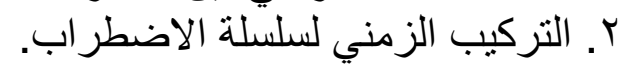
$Y_{t}=c+v(B) X_{t}+N_{t}$ وبذلك يأخذ نموذج الانحدار الثكل الآتي: لئل

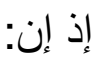

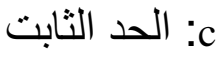
$\mathrm{v}(\mathrm{B})=\mathrm{v}_{0}+\mathrm{v}_{1} \mathrm{~B}+\mathrm{v}_{2} \mathrm{~B}^{2}+\cdots$ . N : سلسلة الخطأ العشو ائي :

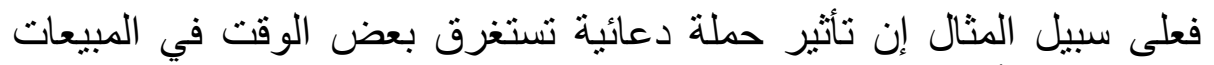

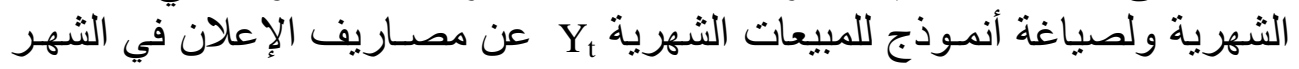


$(r \cdot)^{9} \cdot$

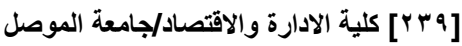

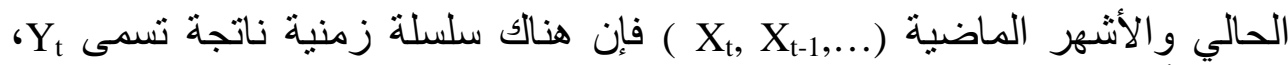

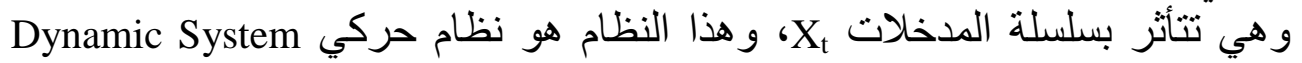

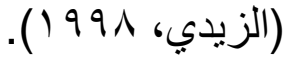

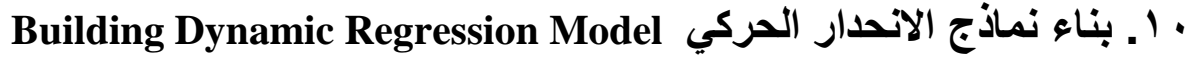
تم ايجاز مراحل بناء نماذج الآج الانحدار الحركي في المخطط الآتي: :(Pankratiz,1991, 174)

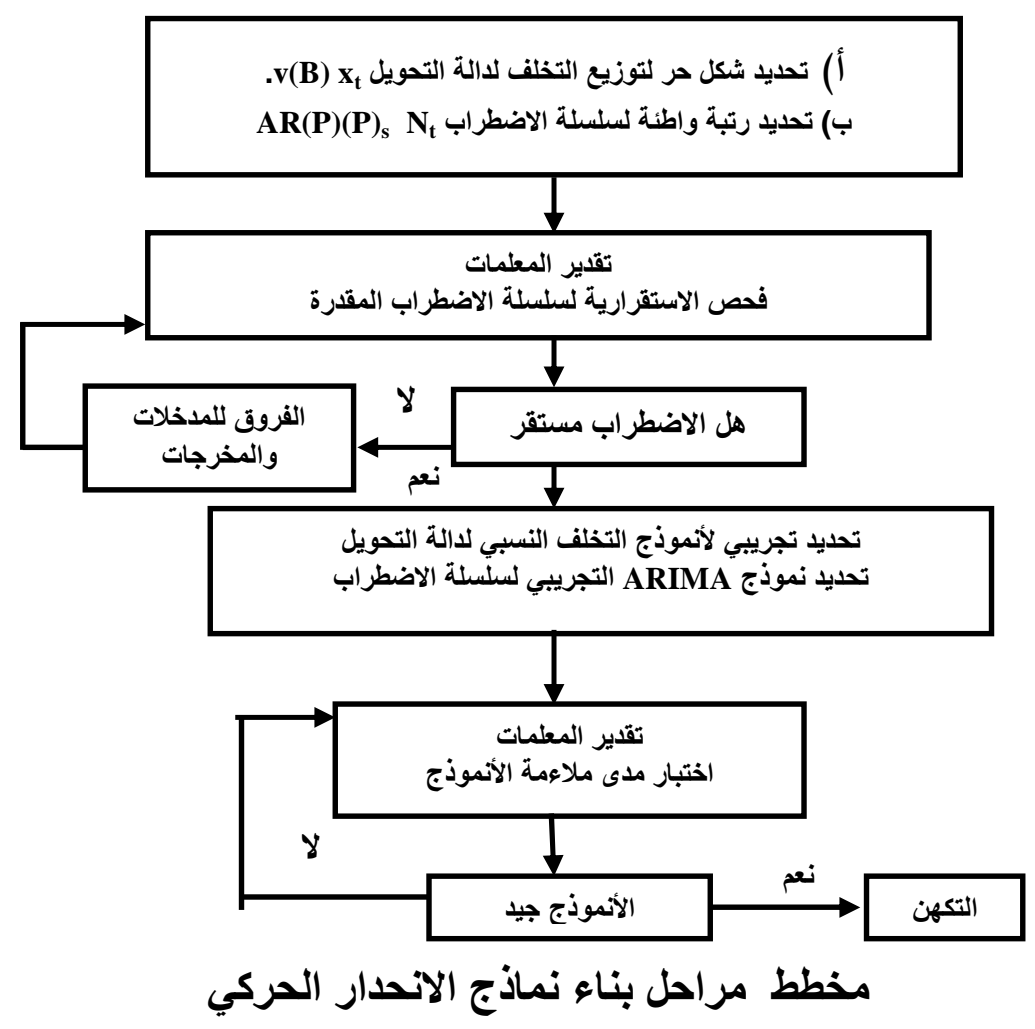

يتم تطبيق خطوات بناء أنموذج الانحدار الحركي على سلسلة معدلات الأمطار

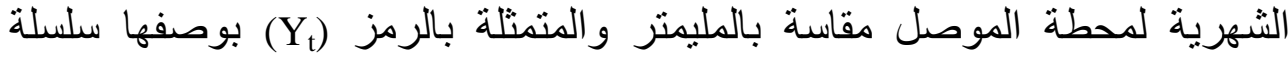

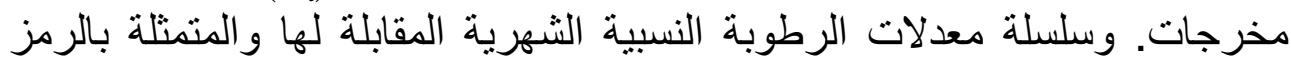
(X)

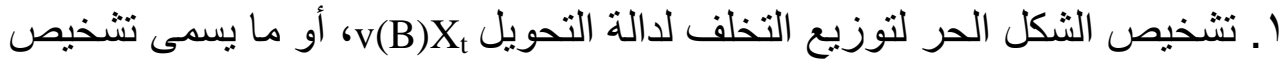
دالة التحويل الخطية Linear Transfer Function) LTF). ولتطبيق طريقة التشخيص (LTF) سلسلة الأمطار وسلسلة الرطوبة النسبية تم أخذ 10=10 ولتقويض الاضطر اب بـ AR فإنه تم أخذ P=1 P=1 بداية (الموسمية وغير الموسمية) و عليه فالأنموذج يكون: الافيطري 


$$
Y_{t}=C+V_{0} X_{t}+V_{1} X_{t-1}+\ldots+V_{10} X_{t-10}+\frac{1}{\left(1-\phi B_{1}\right)\left(1-\phi_{8} B^{8}\right)} a_{t}
$$

وان نتائج تقدير هذه المعادلة موضحة في الجدول الآتي:

\begin{tabular}{|c|c|c|c|c|c|}
\hline \multicolumn{6}{|c|}{ نتائج تقدير معادلة دالة التحويل الخطية } \\
\hline $\mathrm{k}$ & $\hat{\mathrm{v}}_{\mathrm{k}}$ & t & $\mathrm{K}$ & $\hat{\varphi}$ & $|\mathrm{t}|$ \\
\hline 0 & $2<827$ & 13.73 & 1 & 0.438 & 0.3981 \\
\hline 1 & -0.797 & 3.54 & 2 & 9.5 & 8.3 \\
\hline 2 & 0.181 & 0.80 & & & \\
\hline 3 & -0.109 & 0.49 & & & \\
\hline 4 & 0.231 & 1.03 & & & \\
\hline 5 & -0.555 & 2.48 & & & \\
\hline 6 & 0.132 & 0.60 & & & \\
\hline 7 & -0.265 & 1.18 & & & \\
\hline 8 & -0.299 & 1.33 & & & \\
\hline 9 & -0.061 & 0.27 & & & \\
\hline 10 & 0.488 & 2.17 & & & \\
\hline
\end{tabular}

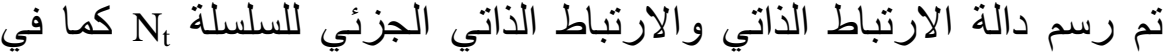

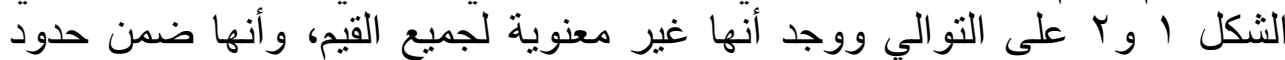

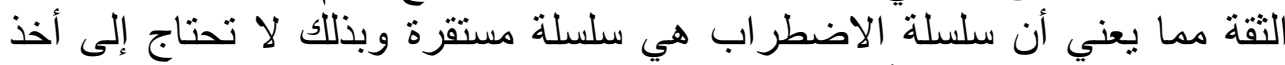
الفروق لسلسلة المدخلات أو سلسلة المخرجات.

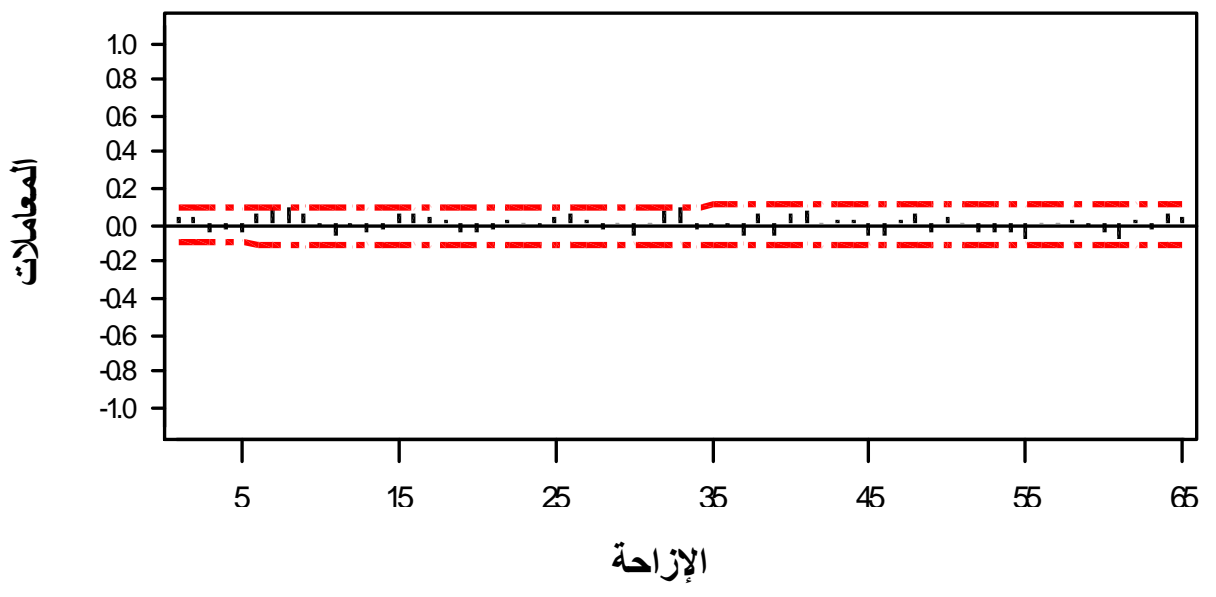

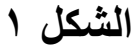

LTF دالة الارتباط الذاتي لسلسلة الأخطاء لأنموذج 
$(r \cdot)^{9} \cdot$

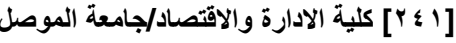

1 ا تحديد رتبة دالة التحويل النسبية

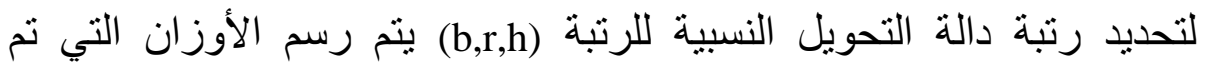
الحصول عليها من تقدير معادلة LTF مع الإزاحة Lag ، وكما موضحة في الثئل الثكل

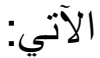

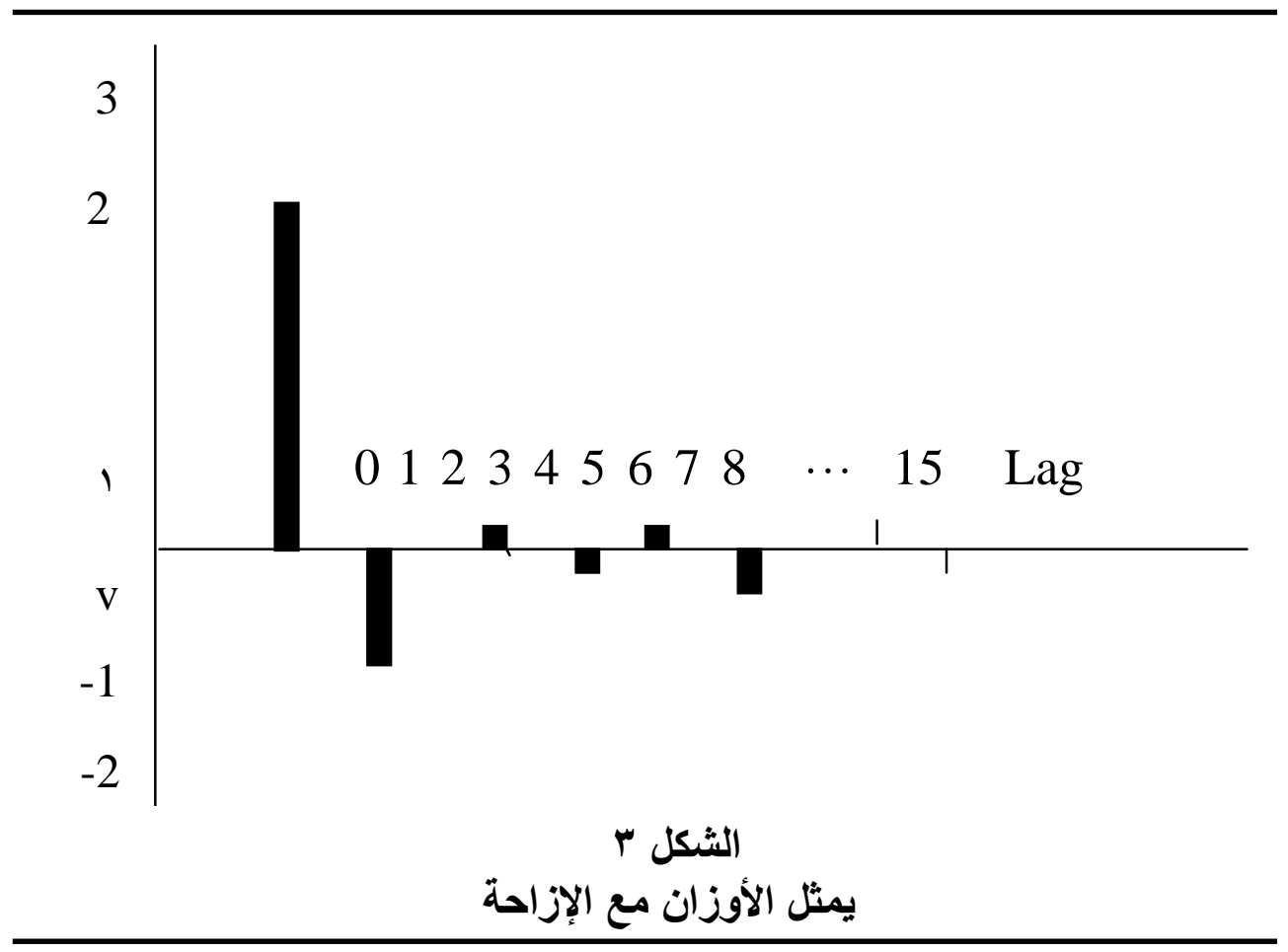

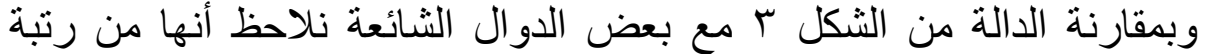

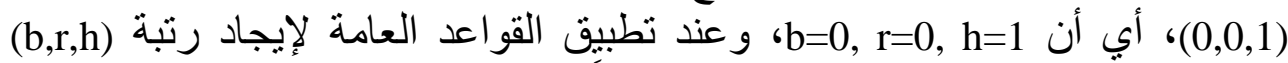

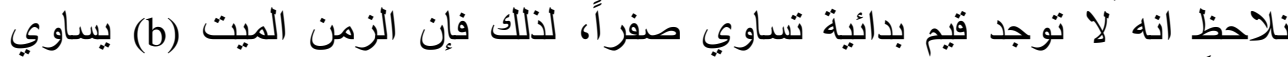

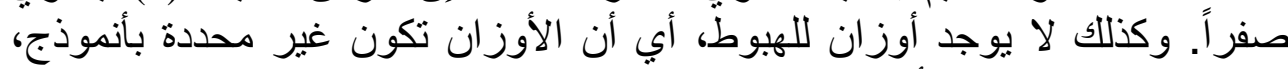

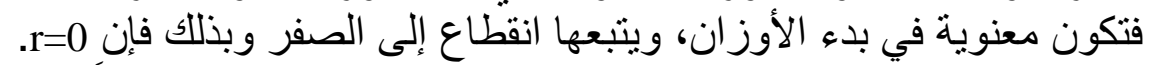

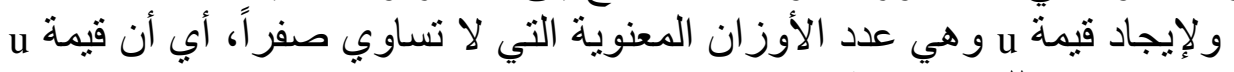

$$
\begin{aligned}
\mathrm{h} & =\mathrm{u}+\mathrm{r}-1 \\
& =2+0-1 \\
& =1
\end{aligned}
$$

وفضلاً عن تحديد أنموذج الصيغة النسبية للأوزان v لابد من تحديد أنموذج

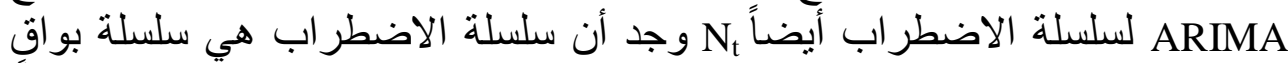
عشوائية أي أن Ne

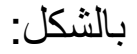




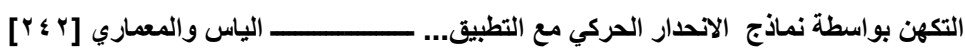

$$
\begin{aligned}
& \mathrm{Y}_{\mathrm{t}}=\left(\mathrm{w}_{0}+\mathrm{w}_{1} \mathrm{~B}\right) \mathrm{X}_{\mathrm{t}}+\mathrm{a}_{\mathrm{t}} \\
& \text { إن أنموذج الانحدار الحركي الدقدر يكون بالصيغة } \\
& \mathrm{y}_{\mathrm{t}}=-116.4757+2.010 \mathrm{x}_{\mathrm{t}}-0.484 \mathrm{x}_{\mathrm{t}-1}+\mathrm{a}_{\mathrm{t}} \\
& \begin{array}{lll}
(-7.3211) & (15.26) & (-3.6349)
\end{array}
\end{aligned}
$$

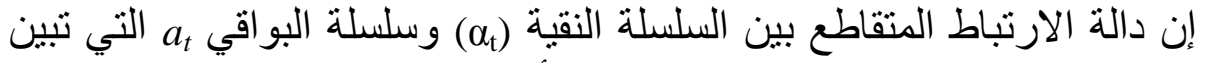

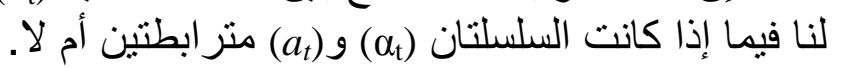
وباستخدام الاختبار الإحصائي (Ljung-Box):

$$
\begin{aligned}
\mathrm{S}^{*} & =\mathrm{n}^{2} \sum_{\mathrm{k}=0}^{\mathrm{K}}(\mathrm{n}-\mathrm{k})^{-1}\left(\mathrm{r}_{\mathrm{k}}^{*}\right)^{2} \\
& =39.8
\end{aligned}
$$

وبمقارنة قيمة الاختبار مع القيمة الجدولية بدرجة حرية 28 وبمستوى معنوية

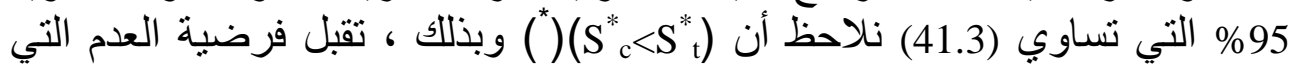
تشير إلى عدم تر ابط السلسلة $a^{2}$

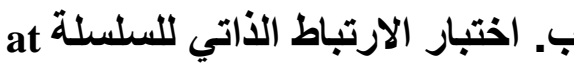

$$
\begin{aligned}
& \text { وباستخدام الاختبار الإحصائي الآتي: } \\
& \text { إختبار (Ljung-Box): } \\
& \varphi^{*}=\mathrm{n}(\mathrm{n}+2) \sum_{\mathrm{k}=1}^{\mathrm{K}}(\mathrm{n}-\mathrm{k})^{-1} \mathrm{r}_{\mathrm{k}}^{2}(\hat{\alpha}) \\
& =46.1
\end{aligned}
$$

وبمقارنة قيمة الاختبار مع القيمة الجدولية بدرجة حرية (65) وبمستوى معنوية

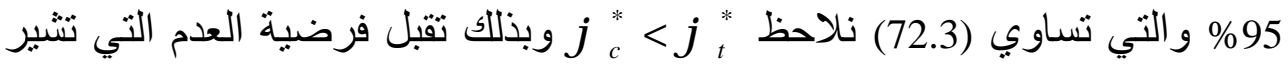
إلى كون سلسلة البو اقي هي سلسلة أخطاء عشو ائية غير مترابطة.

سيتم الحصول على قيم التكهن لأنموذج الانحدار الحركي من خلال الاعتماد

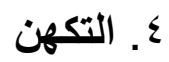

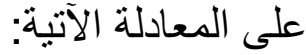

$$
\text { (") القيم بين الأقو اس تمثل قيم t الدحسوبة لمعلمات النموذج. }
$$


$(r \cdot)^{9} \cdot$

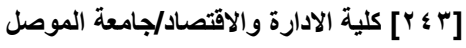

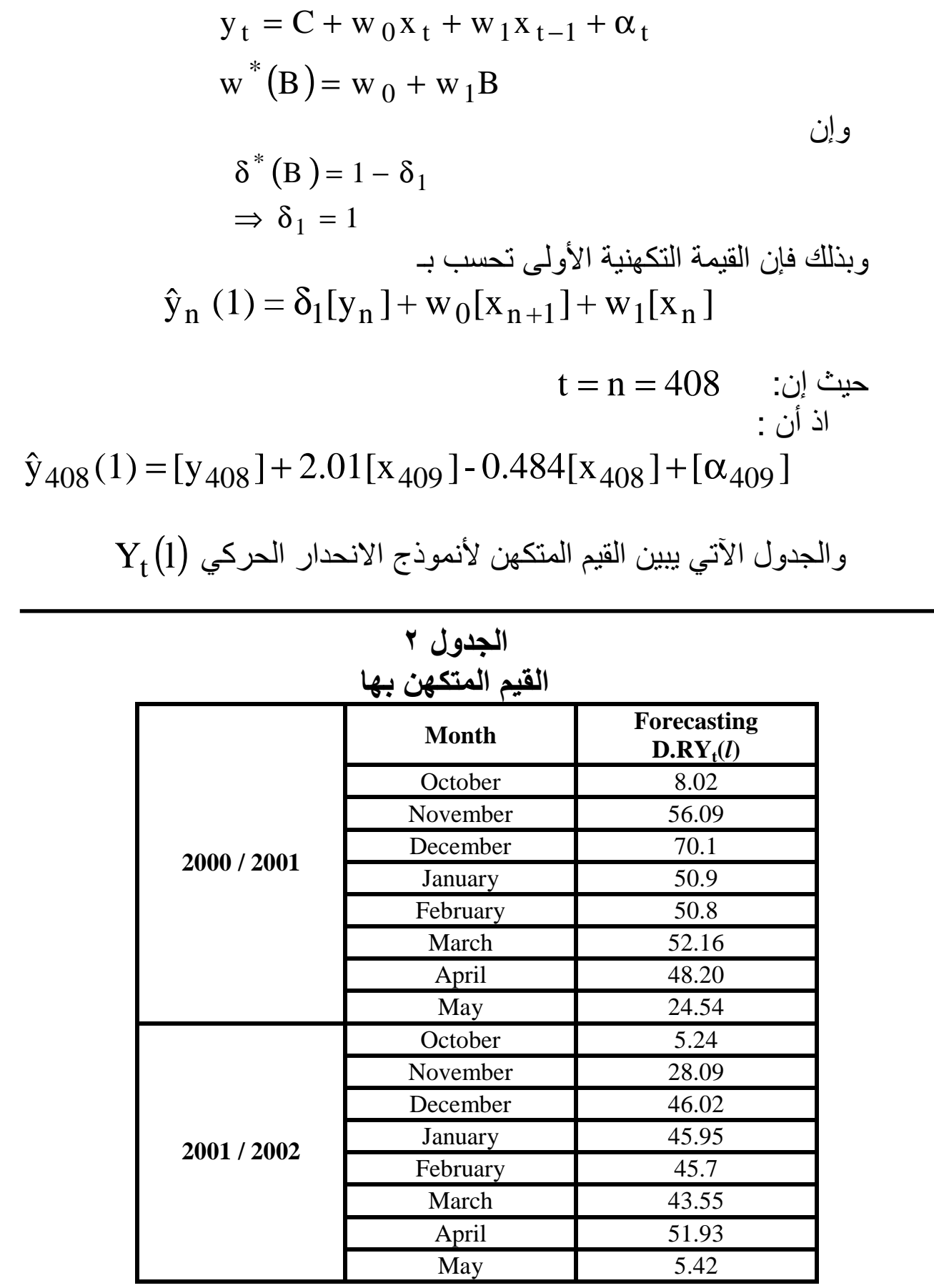

الاستتتاجات Conclusions 


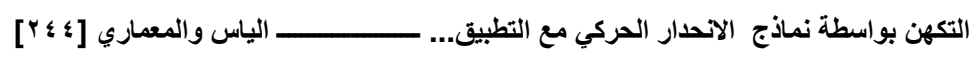

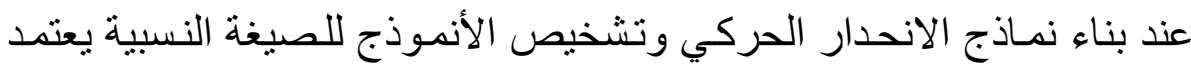

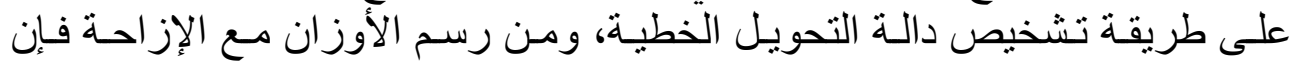

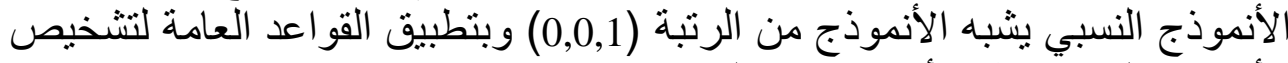
الأنموذج النسبي بظهر أنه كان من الرنبية الأنس نفسها:

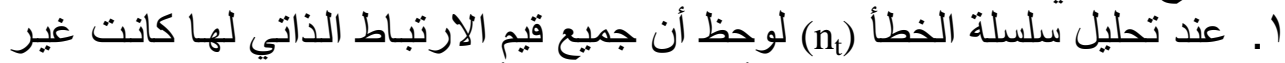

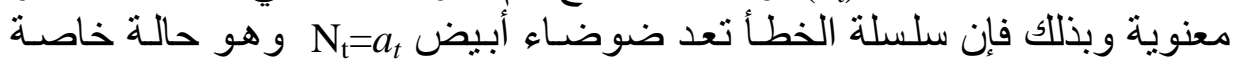

$$
\text { r. الأنموذج الحركي الاتحدار الحركي. للبيانات يكون بالصيغة: }
$$

$$
\begin{aligned}
& \mathrm{Y}_{\mathrm{t}}=\mathrm{C}+\left(\mathrm{w}_{0}+\mathrm{w}_{1} \mathrm{~B}\right) \mathrm{X}_{\mathrm{t}}+a_{t} \\
& \mathrm{Y}_{\mathrm{t}}=-116.4757+2.010 \mathrm{X}_{\mathrm{t}}-0.484 \mathrm{X}_{\mathrm{t}-1}+a_{t} .
\end{aligned}
$$

\section{أولاًا المراجع باللغة العربية}

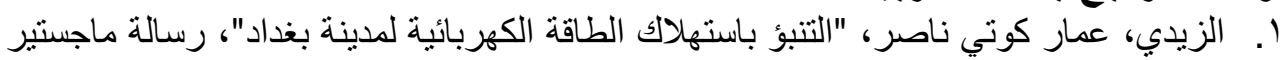

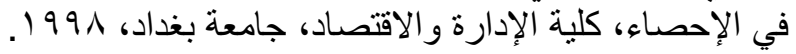

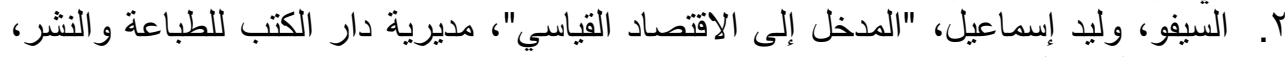

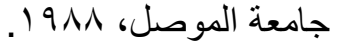

r. الثيخلي، إيفان علاء كاظم، "تصميم نظام رياضي ديناميكي لا خطي باستخدام الثبكات

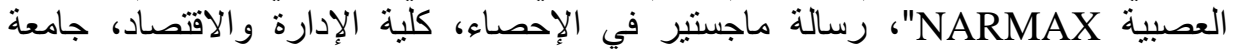

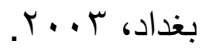

\section{ثانياً. المراجع باللغة الاجنبية}

1.Box, G.E.P. and Jenkins., G.M. "Time Series Analysis Forecasting and Control", San Francisco: Holden-Day, 1976.

2. Makridakis, S. and Whelwright, S.C. "Forecasting Methods and Application", JohnWiley and Sons. Inc, 1978.

3. Pankratz, A., "Forecasting with Dynamic regression Models", John-Wiley and Sons. Inc, 1991. 